\title{
Electronic spectra of carbon chains and rings: Astrophysical relevance?
}

\author{
Evan B. Jochnowitz and John P. Maier \\ Department of Chemistry, University of Basel \\ Klingelbergstrasse 80, Basel, Switzerland \\ email: j.p.maier@unibas.ch
}

\begin{abstract}
Our research has focused on the measurement of the electronic spectra of unstable molecules which are presumed to be of relevance to astrophysical observations. Among these are the carbon chains and their ions. Thus we have been using and developing a number of spectroscopic methods to determine their spectra in the gas phase, including absorption via cavity ring-down and REMPI methods. The species are produced in supersonic jets coupled with discharge and laser ablation sources. With the successful laboratory detection of the electronic spectra of a number of key species, such as bare carbon chains $\mathrm{C}_{n} n=4,5$, comparisons with astrophysical data could be made which lead to interesting implications for the future search for the species which could be responsible for the diffuse interstellar bands. Among the recent relevant observations in the laboratory have been the electronic spectra of carbon rings, $\mathrm{C}_{n}$ $n=14,18,22$, the development of a method to study transitions in mass-selected ions collisionally relaxed to $20 \mathrm{~K}$ and held in a 22 -pole radiofrequency trap, and the study of metal containing carbon chains.
\end{abstract}

Keywords. Astrochemistry, line: identification, molecular data, methods: laboratory, techniques: spectroscopic, ISM: abundances, ISM: clouds, ISM: molecules

\section{Introduction}

There are compelling reasons to obtain the gas-phase electronic spectra of larger carbon chains and rings. This is a direct consequence of studies concerning the absorptions of smaller carbon chains comprising up to a handful of atoms $\mathrm{C}_{n} n=3-5$, simple derivatives $\mathrm{C}_{n} \mathrm{H} n=3-10$, and selected ions $\mathrm{HC}_{2 n} \mathrm{H}^{+} n=2-6, \mathrm{HC}_{2 n} \mathrm{CN}^{+} n=2$, and $\mathrm{NC}_{2 n} \mathrm{CN}^{+}$ $n=1,2$ (Jochnowitz \& Maier 2008). With the laboratory gas-phase spectra in hand, comparisons with astronomical observations were undertaken (Motylewski et al. 2000). Because the selected species given above have absorptions in the 400-1,000 nm region of the diffuse interstellar bands (DIBs) (Snow \& McCall 2006), it proved possible to test directly for the first time the proposition that carbon chains of the size $\mathrm{C}_{n} n=5-10$ are good candidates as carriers (Douglas 1977). Such comparisons showed that these, and related molecules such as polar chains with hydrogen $\mathrm{C}_{n} \mathrm{H}$, can not be responsible for the stronger DIBs (Maier et al. 2004). One expects that in due course they should be observed as very weak DIBs, with equivalent widths (EW) less than $\mathrm{m} \AA$; after all, some of the smaller species, such as $\mathrm{C}_{3} \mathrm{H}$ and $\mathrm{C}_{3} \mathrm{H}_{2}$, are already identified by mm-wave astronomy in the diffuse region (Lucas \& Liszt 2000).

This situation is exemplified by the detection of the $405 \mathrm{~nm} A^{1} \Pi-X^{1} \Sigma^{+}$transition of $\mathrm{C}_{3}$ in diffuse clouds (Maier et al. 2001). Though the individual rotational lines of this transition can be identified, their EWs are around $0.1 \mathrm{~mA}$. Corresponding measurements using the laboratory electronic absorptions of $\mathrm{C}_{4}$ and $\mathrm{C}_{5}$ in the visible were also made but led to the conclusion that their absorptions lie below current detection limits (Maier 


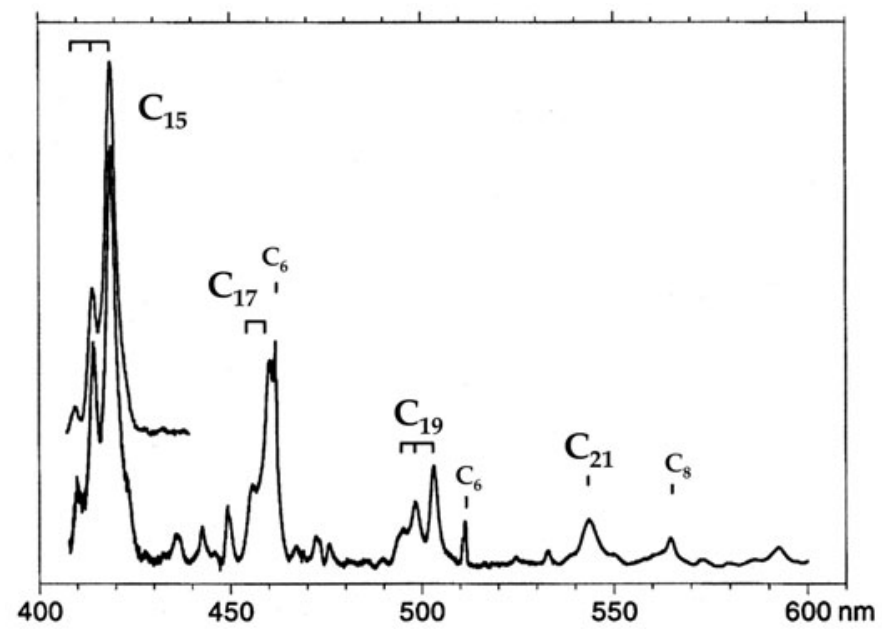

Figure 1. The absorption spectrum of laser-vaporized graphite in a neon matrix. Origin bands belonging to the ${ }^{1} \Sigma_{\mathrm{u}}-X^{1} \Sigma_{\mathrm{g}}^{+}$transition of $\mathrm{C}_{2 n+1} n=7-10$ are identified.

et al. 2002). Same arguments apply to the $\mathrm{C}_{2 n} \mathrm{H}$ series (Motylewski et al. 2000), the latter with $n=1-4$ being well known constituents of dense interstellar clouds with column densities around $10^{12} \mathrm{~cm}^{-2}$. If their column densities are on the order of $10^{11} \mathrm{~cm}^{-2}$ in diffuse clouds then, in view of the oscillator strengths of the electronic transitions (0.05 in magnitude), these would result in DIBs with $\mathrm{m} \AA \mathrm{EW}$ - currently hardly detectable. In fact $\mathrm{C}_{3} \mathrm{H}$ has been detected in the diffuse region with column densities around $10^{11} \mathrm{~cm}^{-2}$ (Lucas \& Liszt 2000). There are several electronic transitions of $\mathrm{C}_{3} \mathrm{H}$ in the visible (Ding et al. 2001), however their absorptions cannot be detected towards reddened stars; their EW are just too small. Similarly, cations such as those of the polyacetylene series, $\mathrm{HC}_{2 n} \mathrm{CN}^{+} n=2$, were chosen for comparison because their neutral species have already been identified in dense clouds. Ions are relevant because it is often argued that the degree of ionization can be high in the diffuse region (Le Page et al. 2003). Furthermore, while the neutrals do not possess electronic transitions in the visible (these lie below $300 \mathrm{~nm}$ ), their ions do (Jochnowitz \& Maier 2008). Thus the hypothesis concerning smaller carbon chains (Douglas 1977) has been tested and can now be rejected (Maier et al. 2004).

\section{Carbon Chains}

However the results point to promising directions for future studies: the longer $\mathrm{C}_{2 n+1} \mathrm{H}$ chains. The reasons for this are: 1) they have electronic transitions in the 400-900 nm region for $n=15-31 ; 2$ ) they possess enormous oscillator strength in the 1-10 range, which implies that column densities of $10^{11} \mathrm{~cm}^{-2}$ would be adequate to give rise to stronger, narrower DIBs with $0.1 \AA \mathrm{EW}$; and 3) these are structurally stable isomers. The absorption spectra of $\mathrm{C}_{2 n+1} n=7-10$ have already been recorded in neon matrices (Wyss et al. 1999), as shown in Figure 1. The transitions are of ${ }^{1} \Sigma_{\mathrm{u}}-X^{1} \Sigma_{\mathrm{g}}^{+}$symmetry, possessing $f>1$ values. The corresponding transition in $\mathrm{C}_{3}$ lies well in the UV, around $170 \mathrm{~nm}$, while the much weaker $A^{1} \Pi_{\mathrm{u}}-X^{1} \Sigma_{\mathrm{g}}^{+}$transitions of the $\mathrm{C}_{2 n+1} \mathrm{H}$ chains shown in Figure 1, lie towards the near IR. Attempts to observe the spectra of longer chains in the gas-phase have failed so far, even though they can be readily produced by laser vaporization sources coupled to supersonic expansions. Figure 2 shows a mass-spectrum of the products in the plasma, obtained by focusing a $7.9 \mathrm{eV}$ laser to generate the ions. 


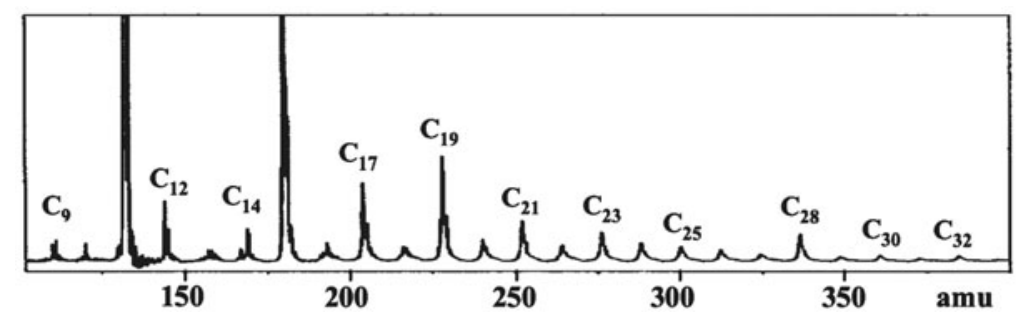

Figure 2. A mass spectrum obtained from the laser ablation of a graphite rod, ionized by a focused $157 \mathrm{~nm}$ laser.

Although the sought species, $\mathrm{C}_{15}, \mathrm{C}_{17}, \mathrm{C}_{19}$, are among the strongest ions, their transitions could not be detected using the resonant 2-color photon ionization (R2CPI) scheme utilizing nanosecond lasers. Though these bare carbon chains satisfy the three criteria given above, it remains to be seen if the excited electronic state $\left({ }^{1} \Sigma_{\mathrm{u}}^{+}\right)$is sufficiently long-lived for these molecules to be spectroscopically relevant. Its lifetime is inferred to be much less than 1 ns because the R2CPI pump-probe excitation failed to detect the transition. If the lifetime is 5 ps then the lifetime broadening will be on the order of $1 \mathrm{~cm}^{-1}$ and the expected rotational contour will still be the major contribution to the total peak width, say a few $\mathrm{cm}^{-1}$, which is comparable to the sharper DIBs with EW of $0.1 \AA$. On the other hand, an excited state lifetime of 0.5 ps leads to a broadening of $10 \mathrm{~cm}^{-1}$, and would therefore not be relevant as carrier of the narrower DIBs. To resolve this dilemna, picosecond pump-probe experiments are in progress in the laboratory.

Independent of this, an appealing, and somewhat unique, aspect of the $\mathrm{C}_{2 n+1}$ chains is that their LUMO $\leftarrow$ HOMO $\pi \leftarrow \pi$ excitations (i.e., corresponding to the absorptions seen in Figure 1) possess large $f$ values. This can be rationalized by consideration of the electronic configuration as described by the $\pi$ molecular orbital occupancies, as shown in Figure 3 where the assorted excitations are demonstrated. For the closed shell $\mathrm{C}_{2 n+1}$ chains it is this excitation which carries the oscillator strength (Figure 3, case I). As for open-shell species, such as carbon chains with even number of atoms or the cations, it is not the lowest energy transition, but rather excitation at higher energy to the LUMO which is the most intense (Figure $3, \mathrm{LUMO} \leftarrow$ HOMO case II and III). This phenomenon has long been known for organic molecules and arises due to configuration mixing.

\section{Carbon Ring Systems}

Using laser vaporization of graphite, together with $1+1^{\prime} \mathrm{R} 2 \mathrm{CPI}$, the electronic spectra of bare carbon species with 14, 18 and 22 carbon atoms could however be observed (Boguslavskiy et al. 2005, Boguslavskiy \& Maier 2007). These follow the Hückel pattern of aromatic molecules. In Figure 4 their spectra are seen, the absorptions spanning the visible to UV region.

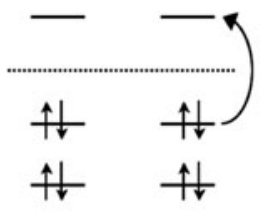

Case I

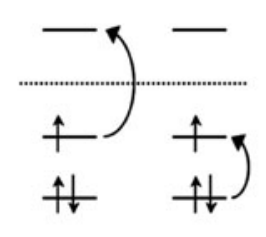

Case II

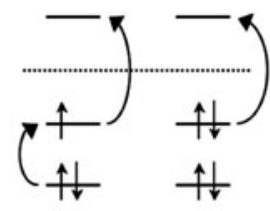

Case III

Figure 3. Types of $\pi-\pi$ excitations encountered in the spectroscopy of carbon chain radicals and ions. 
It should be noted that their lowest electronic transitions fall in the DIB range, the absorptions involve excitation to several electronic states and only the lowest transition demonstrates sufficiently narrow bands $\left(\right.$ few $\mathrm{cm}^{-1}$ ) to be of relevance to the DIB problem. The structures of these bare carbon systems are cyclic. The evidence for this has been presented (Boguslavskiy, Ding, \& Maier 2005), and the study of the linear species in neon matrices shows where they absorb (Wyss et al. 1999). On addition of hydrogen to the plasma the known electronic spectra of the polyacetylene chains $\mathrm{HC}_{2 n} \mathrm{H} n=7,9,11$ (Pino et al. 2001) are observed but their growth does not correlate with the absorptions shown in Figure 4; and the predicted excitation energies (with DFT theory) match reasonably the pattern involving several electronic states. Theory indicates that the cyclic species are stable entities and the geometries predicted are indicated in Figure 4.

The cyclic nature of the molecules is established and is astrophysically relevant. These are the first set of laboratory data on bare carbon cyclic systems in the gas-phase. The position of the origin band of the energetically lowest transition of $\mathrm{C}_{14}, \mathrm{C}_{18}$ and $\mathrm{C}_{22}$ could be compared directly to DIB tabulations or with specific measurements (Maier et al. 2006).

That no DIB absorptions are found at these wavelengths is not surprising in view of criterion 2 presented above; the $f$ value of these transitions is too low; around 0.02 . However the profile of the origin band $(592.8 \mathrm{~nm})$ bears a striking resemblance to DIBs measured at higher resolution (comparable to the laboratory $0.1 \mathrm{~cm}^{-1}$ ), though at different wavelengths (Sarre et al. 1995). This is illustrated in Figure 5. On the left are the

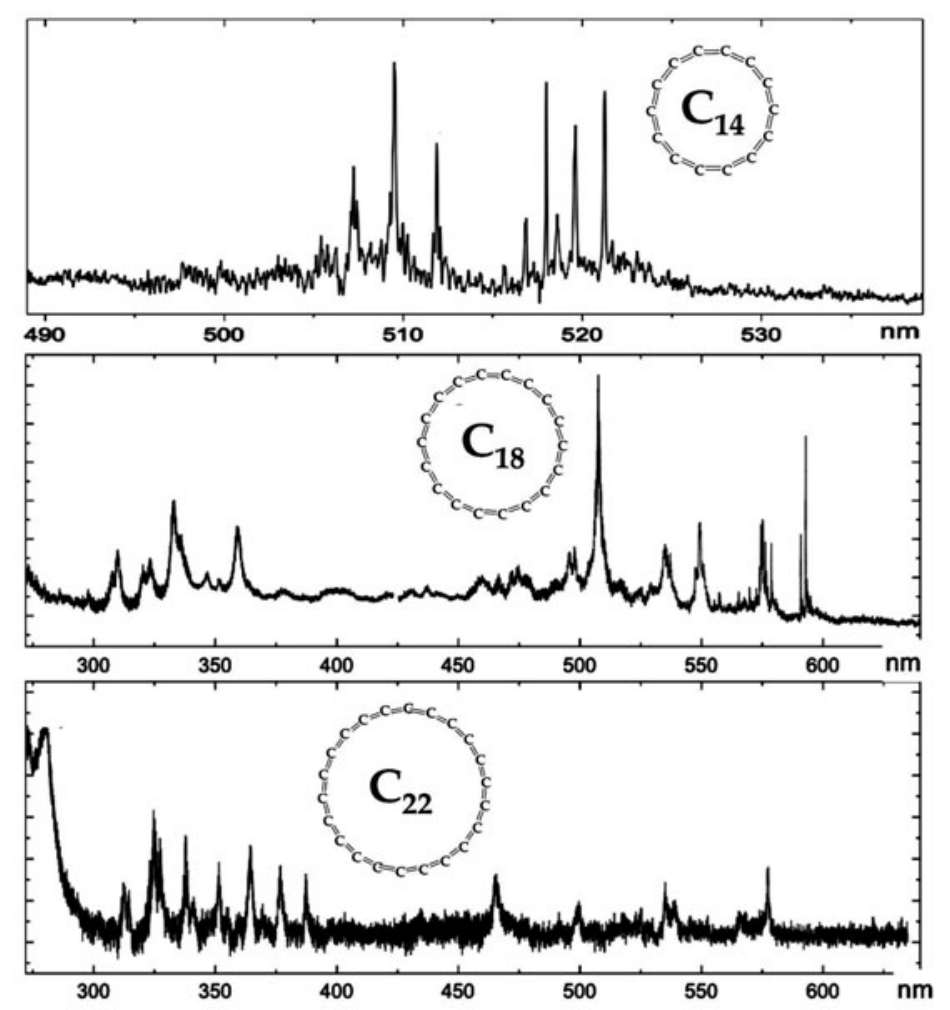

Figure 4. Electronic absorption spectrum of cyclic $\mathrm{C}_{14}, \mathrm{C}_{18}$ and $\mathrm{C}_{22}$ measured in the gas phase using a resonant 2-color photoionization scheme. The spectra show several electronic transitions, with the bands broadening out with increasing energy. 
laboratory spectra at around 100, 50 and $20 \mathrm{~K}$. The changes in intensity of the three peaks apparent are attributed to residual vibrational excitation. Even though the species are produced in supersonic expansions, low frequency vibrations are difficult to cool. The peak to the right is a hot band, as is probably the one to the left of the central one. The latter is the origin band itself, constituting a parallel transition according to the calculated excitation energies, the rotation structure concealed within its $1-2 \mathrm{~cm}^{-1}$ width. The three peak structure of the DIB at $661.4 \mathrm{~nm}$ is almost superimposable with the laboratory spectrum if they are shifted in wavelength above each other. As another example, the twin-peaked DIB at $579.7 \mathrm{~nm}$ (bottom right of Figure 5) can be superimposed on the laboratory spectrum at $20 \mathrm{~K}$. This similarity is so striking that it prompts one to ask if it is not indeed plate-like molecules, comprising say 30-100 carbon atoms, which could be the carriers of the DIBs at 579.7 and $661.4 \mathrm{~nm}$ ?

According to the criteria discussed, these respective molecules would have to have $f$ values around unity to satisfy the abundance constraints. These species may also be neutral or ionic. Relevant for astrophysical considerations is the fact that UV irradiation of middle-sized polyaromatic hydrocarbon cations, e. g., that of coronene cation, $\mathrm{C}_{24} \mathrm{H}_{12}^{+}$, with fluxes similar as found in the interstellar medium, leads to the loss of all $\mathrm{H}$ atoms, leaving behind only the photostable bare carbon ring, $\mathrm{C}_{24}^{+}$(Ekern et al. 1998). So how could one measure the absorption features of species such as $\mathrm{C}_{24}^{+}$in the laboratory?

\section{Molecular Cations}

With this goal we are developing an approach to accomplish this. The principle is to study mass-selected ions held in a radio frequency (rf) trap and cooled by collisions to cryogenic temperatures. The ions have been produced in an electron impact source, mass-selected by a quadrupole selector, and then injected into a 22-pole rf trap. There the vibrational and rotational degrees of freedom are relaxed as a result of collisions with helium gas cooled to $10 \mathrm{~K}$. The electronic transitions are then induced by laser

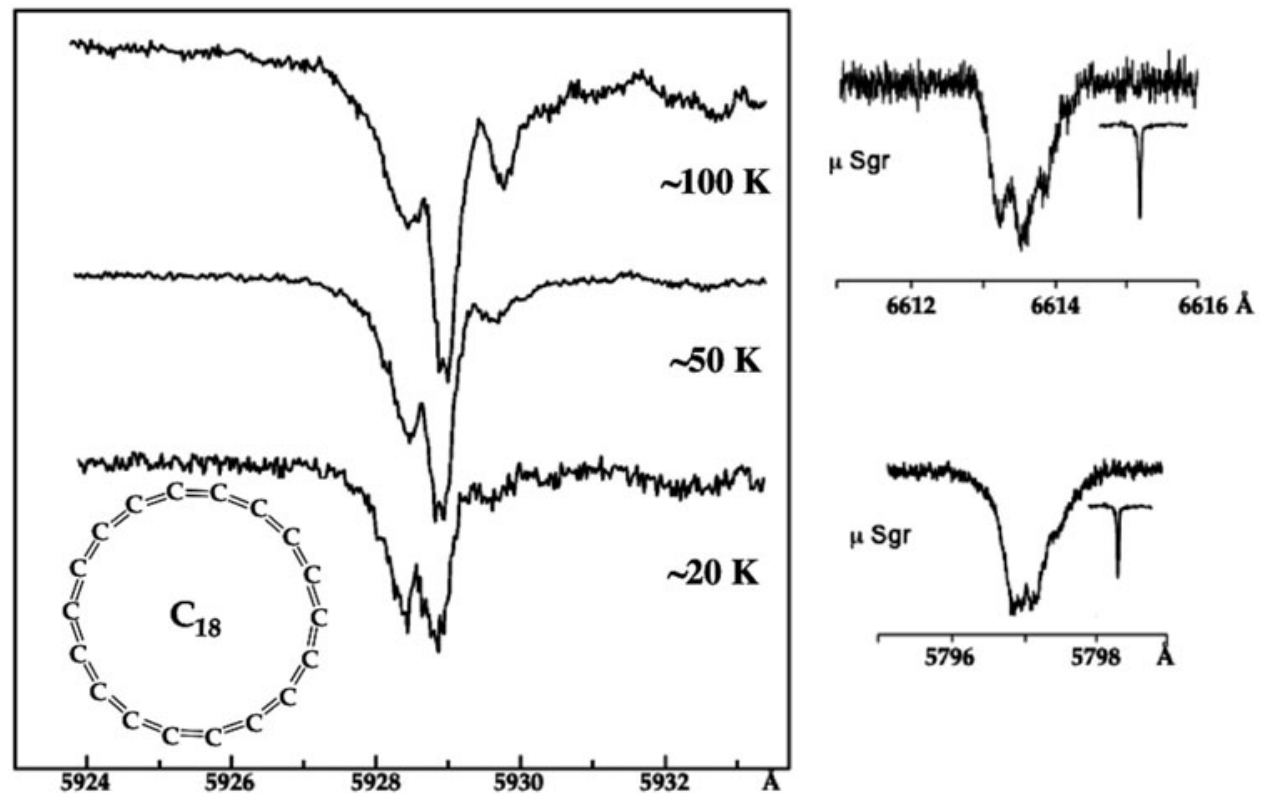

Figure 5. To the left lies the origin band of the first electronic transition of cyclic $\mathrm{C}_{18}$ measured in the gas phase. On the right two different diffuse interstellar absorption features observed towards indicated stars. Reproduced from Sarre et al. (1995). 
photon absorption from the ions in their ground states, cooled to their lowest vibrational level with few rotational levels still populated. Following the population of the excited electronic state after the laser excitation, a second UV laser leads to fragmentation. Afterwards the trap is subsequently opened and the second quadrupole is set to transmit the fragment ions.

The initial experiments with the 2,4-hexadiyne cation, a $\mathrm{C}_{6} \mathrm{H}_{6}^{+}$isomer, proved that both the vibrations and rotations have indeed been cooled to temperatures near $20 \mathrm{~K}$ (Dzhonson \& Maier 2005). As an example for ions of potential astrophysical interest, the electronic spectrum of $\mathrm{HC}_{12} \mathrm{H}^{+}$recorded by this two colour two photon approach (Dzhonson et al. 2007) is shown in Figure 6.

The absorption is to the lowest electronic state, $A^{2} \Pi_{\mathrm{g}}-X^{2} \Pi_{\mathrm{u}}$, with origin band at $924.7 \mathrm{~nm}$. This is the same region where weak DIBs have been located in connection with the search for $\mathrm{C}_{60}^{+}$(Foing \& Ehrenfreud 1994), whose origin band of its lowest electronic transition lies at $964 \mathrm{~nm}$ in a neon matrix (Fulara et al. 1993). The gas phase spectrum, however, is still not available. The inset of Figure 6 shows the gas-phase location of the origin bands of the other members of this homologous series of polyacetylene cations. Because these gas-phase laboratory data are available, a comparison with DIB observations has been made; in all cases only upper limits to column densities could be inferred, generally $<10^{12} \mathrm{~cm}^{-2}$. Though these are the energetically lowest allowed transitions for these ions, with $f$ values around 0.05 , the next $\pi-\pi$ excitation is the much stronger one and appealing for comparison with DIB absorptions. These have been located in neon matrices (Fulara et al. 2007), e.g. near $700 \mathrm{~nm}$ for $\mathrm{HC}_{12} \mathrm{H}^{+}$, but not as yet in the gas phase. It remains to be seen how broad the bands will be as a result of intramolecular processes.

\section{Metal Capped Chains}

Another class of molecules which may have sufficiently large oscillator strengths would be carbon chains containing heavy atoms due to their polarisability. Among these could be those with oxygen, and also the relatively abundant elements $\mathrm{Mg}, \mathrm{Fe}$, and $\mathrm{Al}$. Especially intense will be the charge transfer transitions for heavier metal-capped chains, at wavelengths corresponding to the difference between the ionization potential of the heavy atom and the electron affinity of the carbon chain. The latter can be quite large (3$5 \mathrm{eV}$ ) for the longer carbon chains, placing transitions in the visible spectral range. Thus the measurement of the electronic spectra of carbon chains with these metals present is

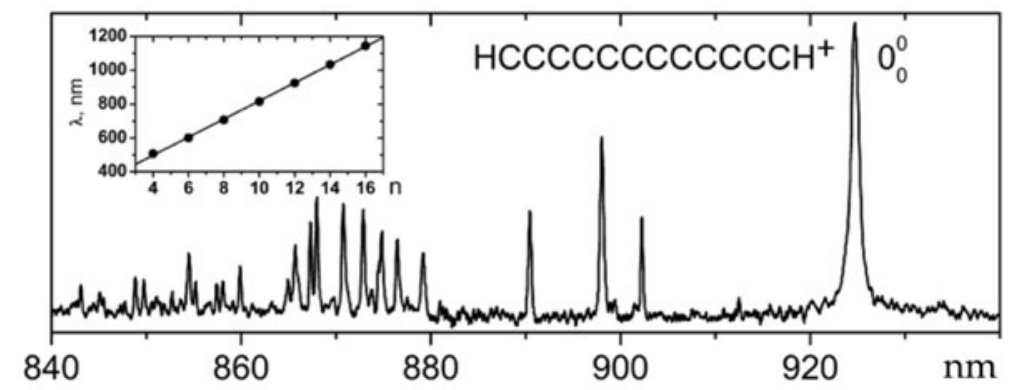

Figure 6. Electronic gas-phase absorption spectrum of the $A^{2} \Pi_{\mathrm{g}}-X^{2} \Pi_{\mathrm{u}}$ transition in $\mathrm{HC}_{12} \mathrm{H}^{+}$ cation, observed using a 2-photon 2-color photofragmentation technique in a 22-pole rf ion trap, where species are vibrationally and rotationally relaxed through collisions with cryogenically cooled helium gas. An inset shows the dependence of the wavelength of absorption with chain size. 


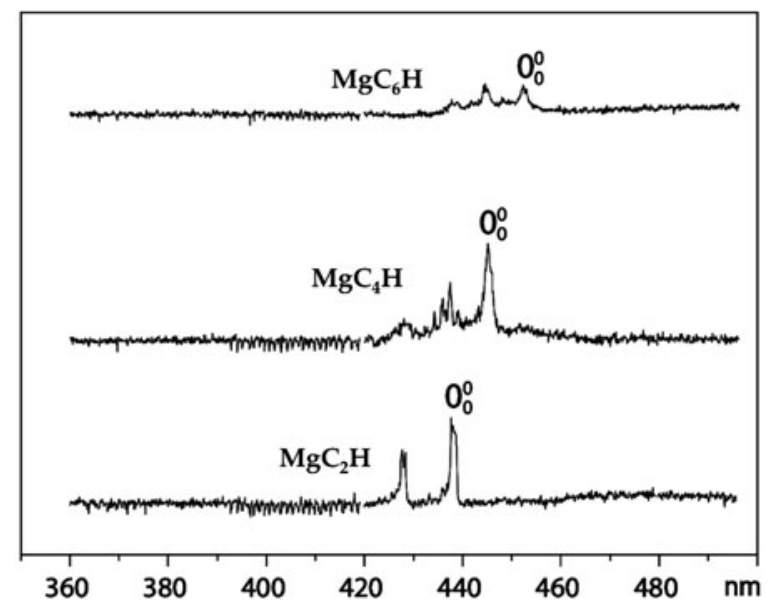

Figure 7. Electronic spectra of the $A^{2} \Pi-X^{2} \Sigma^{+}$transition in $\mathrm{MgC}_{n} \mathrm{H}(n=2,4,6)$, measured in the gas phase using a resonant 2 -color photoionization technique.

being pursued in the laboratory. The first success in this has been the detection of $\mathrm{MgC}_{n} \mathrm{H}$ chains, with $n=4,6$ (Ding et al. 2008), the electronic transition of the species with $n=2$ having being previously observed (Corlett et al. 1996). Their $A^{2} \Pi-X^{2} \Sigma^{+}$transitions lie in the visible and the respective spectra shown in Figure 7 were detected by the R2CPI approach using laser vaporization of a magnesium rod over which acetylene was passed. The systems have moderate oscillator strength, around 0.1 ; the electron excitation is mainly localized on the $\mathrm{Mg}$ atom. In addition the electronic spectra of AlCCH (Apetrei et al. 2007) and $\mathrm{AlC}_{2}$ (Chasovskikh et al. 2007) were recently observed in the gas phase using laser induced fluorescence and R2CPI.

\section{Acknowledgements}

The research is supported by the Swiss National Science Foundation (project 200020$115864 / 1)$.

\section{References}

Apetrei, C., Ding, H., \& Maier, J. P. 2007, Phys. Chem. Chem. Phys., 9, 3897

Boguslavskiy, A. E., Ding, H., \& Maier, J. P. 2005, J. Chem. Phys., 123, 034305

Boguslavskiy, A. E. \& Maier, J. P. 2007, Phys. Chem. Chem. Phys., 9, 127

Chasovskikh, E., Jochnowitz, E. B., Kim, E., Maier, J. P., \& Navizet, I. 2007, J. Phys. Chem. $A, 111,11986$

Corlett, G. K., Little, A. M., \& Ellis, A. M. 1996, Chem. Phys. Lett, 249, 53

Ding, H., Apetrei, C., Chacaga, L., \& Maier, J. P. 2008, ApJ, 677, 348

Ding, H., Pino, T., Guethe, F., \& Maier, J. P. 2001, J. Chem. Phys., 115, 6913

Douglas, A. E. 1977, Nature, 269, 130

Dzhonson, A., Jochnowitz, E. B., \& Maier, J. P. 2007, J. Phys. Chem. A, 111, 1887

Dzhonson, A. \& Maier, J. P. 2005, Int. J. Mass. Spectrom., 255, 139

Ekern, S. P., Marshall, A. G., Szczepanski, J., \& Vala, M. 1998, J. Phys. Chem. A, 102, 3498

Foing, B. \& Ehrenfreud, P. 1994, Nature, 369, 296

Fulara, J., Grutter, M., \& Maier, J. P. 2007, J. Phys. Chem. A, 111, 11831

Fulara, J., Jakobi, M., \& Maier, J. P. 1993, Chem. Phys. Lett., 211, 227

Jochnowitz, E. B. \& Maier, J. P. 2008, Annu. Rev. Phys. Chem., 59, 519

Le Page, V., Snow, T. P., \& Bierbaum, V. M. 2003, ApJ, 584, 316

Lucas, R. \& Liszt, H. 2000, A\&A, 358, 1069 
Maier, J. P., Boguslavskiy, A. E., Ding, H., Walker, G. A. H., \& Bohlender, D. A. 2006, ApJ, 640, 369

Maier, J. P., Lakin, N. M., Walker, G. A. H., \& Bohlender, D. A. 2001, ApJ, 553, 267

Maier, J. P., Walker, G. A. H., \& Bohlender, D. A. 2002, ApJ, 566, 332

Maier, J. P., Walker, G. A. H., \& Bohlender, D. A. 2004, ApJ, 602, 286

Motylewski, T., Linnartz, H., Vaizert, O., Maier, J. P., Galazutidinov, G. A., Musaev, F. A., Krelowski, J., Walker, G. A. H., \& Bohlender, D. A. 2000, ApJ, 531, 312

Pino, T., Ding, H., Guethe, F., \& Maier, J. P. 2001, J. Chem. Phys., 114, 2208

Sarre, P. J., Miles, J. R., Kerr, T. H., Hibbins, R. E., Fossey, S. J. \& Somerville, W. B. 1995, MNRAS (Letters), 277, L41

Snow, T. P. \& McCall, B. J. 2006, ARAA, 44, 367

Wyss, M., Grutter, M. \& Maier, J. P. 1999, Chem. Phys. Lett., 304, 35

\section{Discussion}

Mulas: These are beautiful measurements. However, I'm doubtful that they can be used for diffuse bands because it looks like, with very few exceptions, all of the bands are due to one carrier. There is no such close correlation of diffuse bands; you can't attribute all the different bands to the same carrier. Most of the beautiful spectra you showed had many strong bands, not just one. This would seem to be difficult to reconcile with observations.

MAIER: This is misleading. Because the way we do the experiments these intensity ratios are not realistic and are artificially distorted. When you do a linear absorption measurement, if this origin band is $100 \%$, the next vibrational excitations are about $10-20 \%$ in intensity.

Mulas: So indeed you do have one strong band and one weak band ...

MAIER: One strong origin and several weaker bands to higher energy is typical for these species.

Mulas: You showed a scheme for the relaxation of these molecules and indicated that many should undergo some fluorescence or luminescence. You showed you expected excitation, then an intramolecular transition, and then some of the energy would be emitted by either infrared or ...

MAIER: Most polyatomic radicals and ions do not relax by fluorescence from their excited electronic states. However, after rapid intramolecular conversion, in the absence of collisions, infrared emission is possible.

Mulas: So, could you in some cases predict something observable in emission for the relaxation? If they absorb energy and re-emit it in space, it could be observable.

MAIER: That's correct. This would be something like a phosphorescence-equivalent processes. The way we do it, we trap the relaxed ions in some sort of a long-lived state. For example, $\mathrm{C}_{5}$ is in a triplet state. We drive the triplet state to the ionization continuum, and that's the way we detect these species. Indeed, this triplet state in interstellar space may well decay by phosphorescence. We know very little about these sorts of transitions as yet. 